Supporting Information:

\title{
How the Natural Organic Matter to Coagulant Ratio Impacts on Floc Structural Properties
}

Peter Jarvis, Bruce Jefferson* and Simon A. Parsons

Pages: 2

Figures: 3

Tables: 0 


\section{Settling Measurement}

The settling column apparatus consisted of a central settling column enclosed by a water bath to ensure a constant temperature of $21-22{ }^{\circ} \mathrm{C}$ during floc settlement. The settling column was filled with de-ionized water and left to reach the required temperature. A period of 2 hours was left for quiescent conditions to be reached and the temperature to stabilize. Floc aggregates were introduced into the settling column via a tapered entry port to ensure the flocs settled into the centre of the column using a wide mouthed pipette. Floc images were captured using a CV M90 color chargecoupled device (CCD) camera (JAI UK Ltd, England). Image analysis software (Image Pro Plus from Media Cybernetics, Maryland, USA) was used to determine floc settling velocity and had been calibrated using a microscope graticule.

Flocs were aggregated on a jar tester under the required conditions at room temperature (21-22 ${ }^{\circ} \mathrm{C}$ ). Flocs were then carefully removed from the jar using a wide mouthed pipette and re-suspended in de-ionized water held in a water bath at $21-22{ }^{\circ} \mathrm{C}$ for dilution and temperature control of the flocs. Flocs were then introduced into the settling column. Visual observation showed that little change in floc macrostructure occurred using the pipettes, however if flocs were observed to break during the transfer procedure then they were discarded. Using the described protocol ensured that flocs were able to settle in relative isolation of one another to prevent hindered settling effects.

As a focused floc passed in front of the camera, the image grabber was manually triggered to take a series of 5 images. The time between each frame was set at 1 second. This meant that the distance traveled by the floc could be calculated per frame and therefore per time period, thus giving a settling velocity. The projected area of the floc presented in front of the camera was determined using the image analysis software and converted to an equivalent diameter. This standardized diameter was recorded along with its settling velocity for approximately 100 aggregates for each set of coagulation conditions. 


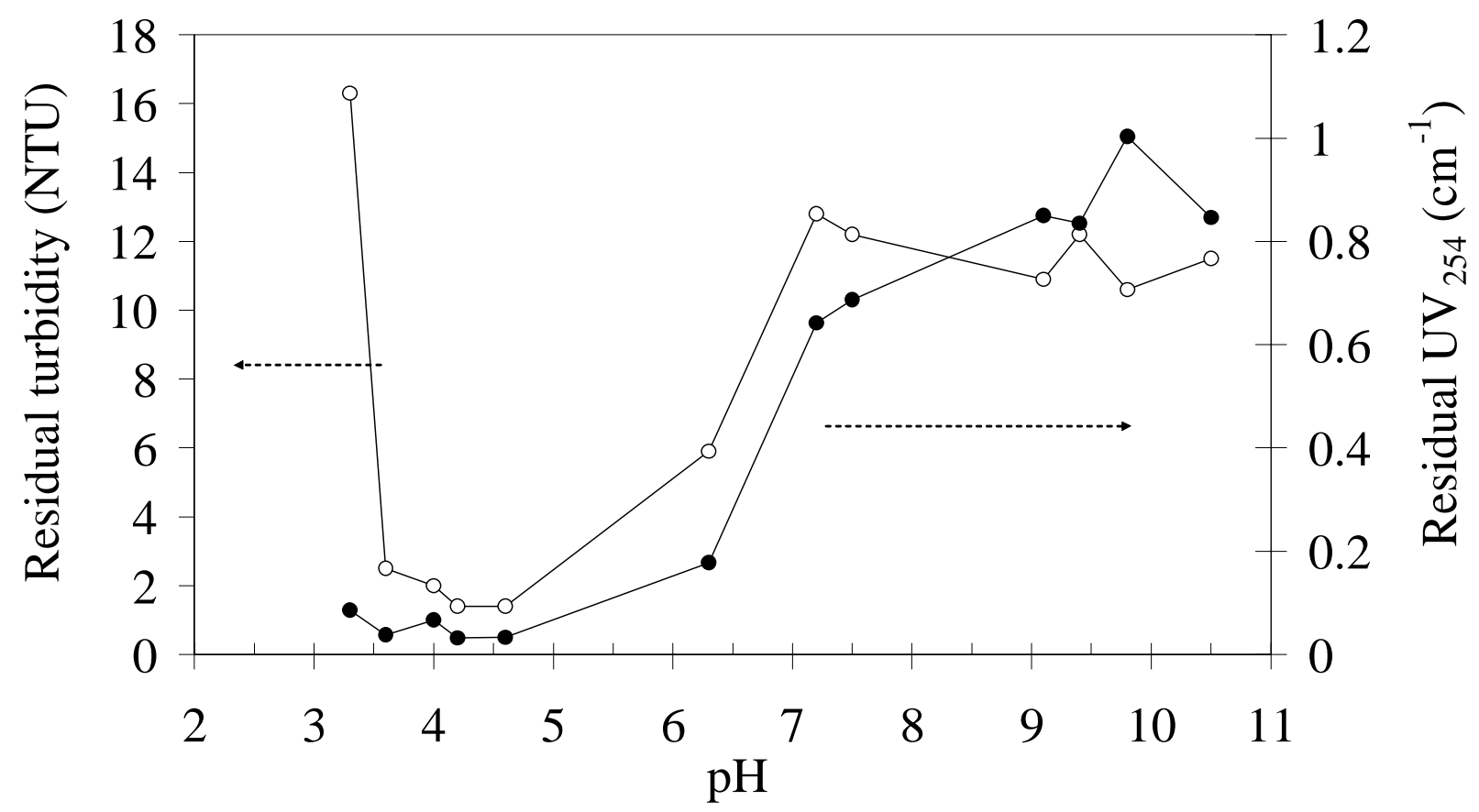

Figure I. Optimization of NOM removal with $\mathrm{pH}$ for a coagulant dose of $8 \mathrm{mg} \mathrm{L}^{-1}$ as $\mathrm{Fe}$ for $\mathrm{UV}_{254}$ and turbidity on the raw water (ratio 1:1). 


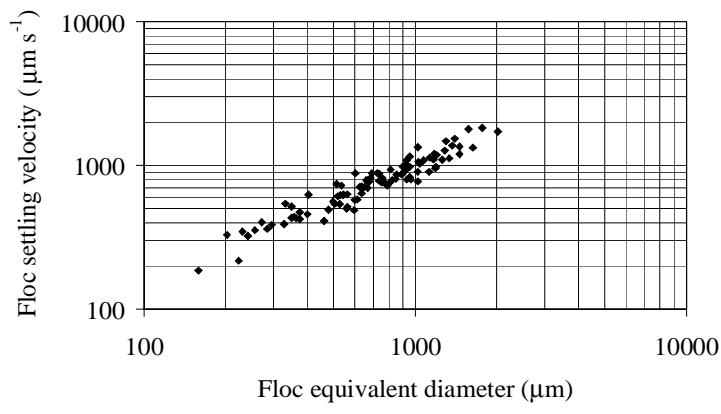

Ratio: 0 (Precipitate)

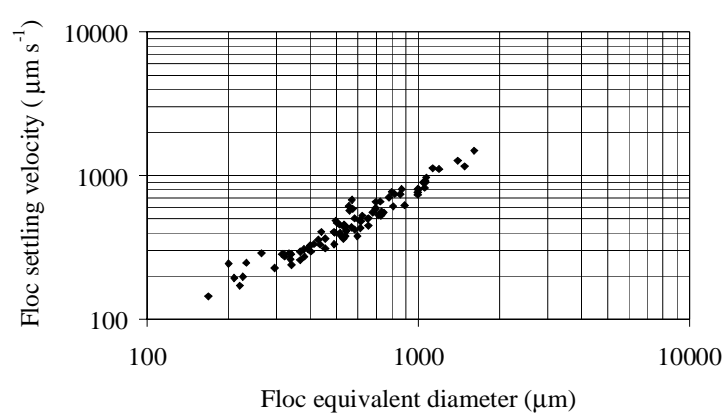

Ratio: 0.75

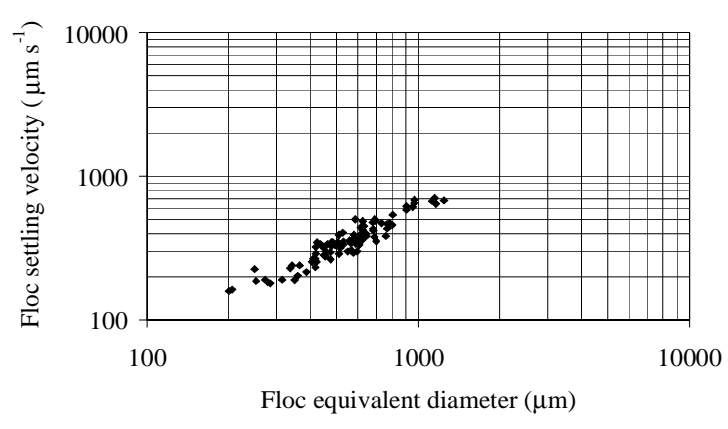

Ratio: 1.75

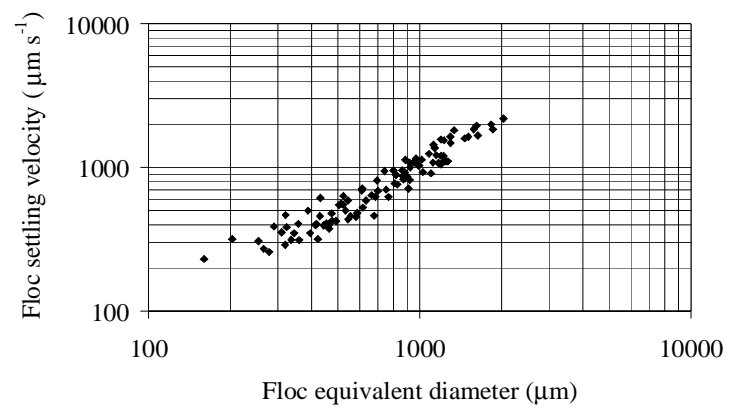

Ratio: 0.33

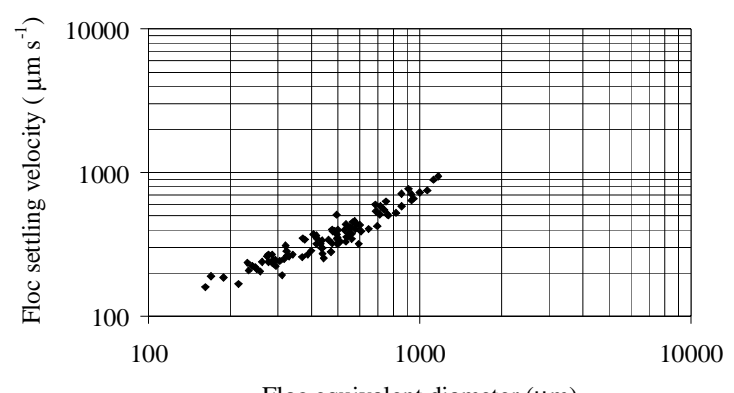

Floc equivalent diameter $(\mu \mathrm{m})$

\section{Ratio: 1}

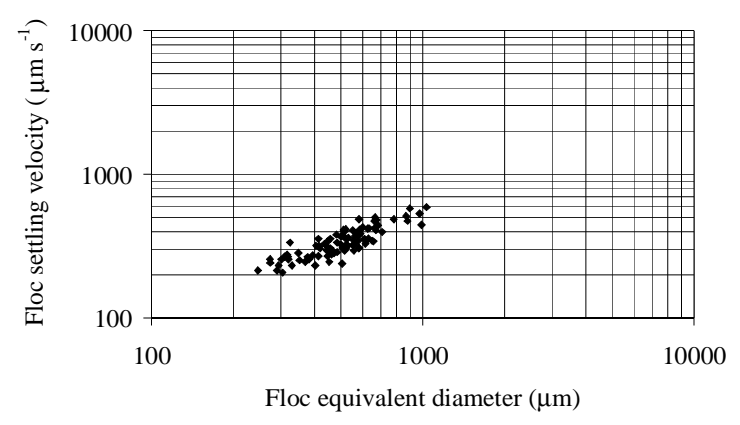

Ratio: 3.8

Figure II. The settling velocity of each of the different DOC:Fe ratio flocs. 


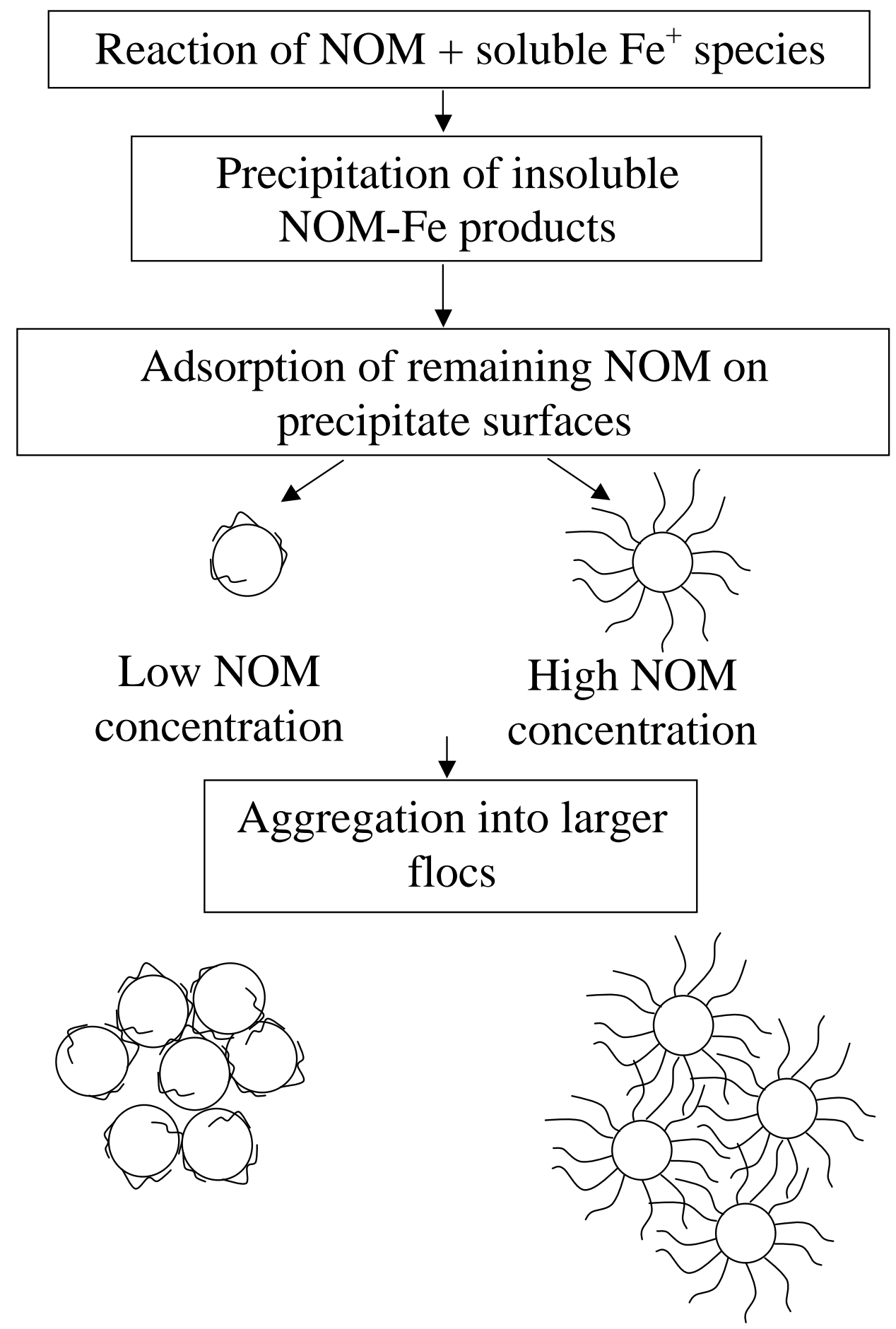

Figure III. The proposed model for the formation of flocs at low and high DOC:Fe ratios. 